\title{
CNS/PNS Boundary Transgression by Central Glia in the Absence of Schwann Cells or Krox20/Egr2 Function
}

\author{
Fanny Coulpier, ${ }^{1,2}$ Laurence Decker, ${ }^{2}$ Benoît Funalot, ${ }^{3,4}$ Jean-Michel Vallat, ${ }^{4}$ Federico Garcia-Bragado, ${ }^{5}$ \\ Patrick Charnay, ${ }^{2}$ and Piotr Topilko ${ }^{2}$ \\ 1Plate-forme Transcriptome and 2Developmental Biology Section, Inserm, Unité 1024, Centre National de la Recherche Scientifique, Unité Mixte de \\ Recherche 8197, Institut de Biologie de l'Ecole Normale Supérieure, 75230 Paris, France, ${ }^{3}$ Laboratoire de Biochimie et Génétique Moléculaire and ${ }^{4}$ National \\ Reference Center "Rare Peripheral Neuropathies," Department of Neurology, Centre Hospitalier Universitaire de Limoges, 87042 Limoges, France, and \\ ${ }^{5}$ Servicio de Anatomia Patologica, Hospital Virgen del Camino, 31008 Pamplona, Spain
}

CNS/PNS interfaces constitute cell boundaries, because they delimit territories with different neuronal and glial contents. Despite their potential interest in regenerative medicine, the mechanisms restricting oligodendrocytes and astrocytes to the CNS and Schwann cells to the PNS in mammals are not known. To investigate the involvement of peripheral glia and myelin in the maintenance of the CNS/PNS boundary, we have first made use of different mouse mutants. We show that depletion of Schwann cells and boundary cap cells or inactivation of Krox20/Egr2, a master regulatory gene for myelination in Schwann cells, results in transgression of the CNS/PNS boundary by astrocytes and oligodendrocytes and in myelination of nerve root axons by oligodendrocytes. In contrast, such migration does not occur with the Trembler ${ }^{J}$ mutation, which prevents PNS myelination without affecting Krox20 expression. Altogether, these data suggest that maintenance of the CNS/PNS boundary requires a Krox20 function separable from myelination control. Finally, we have analyzed a human patient affected by a congenital amyelinating neuropathy, associated with the absence of the KROX20 protein in Schwann cells. In this case, the nerve roots were also invaded by oligodendrocytes and astrocytes. This indicates that transgression of the CNS/PNS boundary by central glia can occur in pathological situations in humans and suggests that the underlying mechanisms are common with the mouse.

\section{Introduction}

In vertebrates the CNS and PNS are segregated at both anatomical and cellular levels. They interface at the level of specific zones on the CNS surface, in which motor axons exit and sensory axons enter the CNS. These interfaces constitute cell boundaries, because they delimit territories with different neuronal and glial contents. The main glial components are astrocytes and oligodendrocytes in the CNS and Schwann cells in the PNS, and the CNS/PNS boundary is characterized by a tight apposition between astrocytes and Schwann cells (Berthold and Carlstedt, 1977). Although Schwann cells and oligodendrocytes are both in charge of axon myelination (Sherman and Brophy, 2005), they have distinct morphological and molecular properties and different embryonic origins, the neural crest and the neural tube, re-

\footnotetext{
Received Jan. 4, 2010; revised March 9, 2010; accepted March 17, 2010.

This work was supported by the Institut National de la Santé et de la Recherche Médicale and by grants from the Ministère de l'Education Nationale et de la Recherche Technologique, Agence Nationale pour la Recherche Grant ANR-05-NEUR-022, the Association pour la Recherche sur le Cancer, the Association Française contre les Myopathies, and the Association Européenne contre les Leucodystrophies. We are grateful to P. Soriano, S. Dufour, and B. Crenshaw for the Rosa26 ${ }^{\text {lacZ }}$, HtPA:Cre, and Brn4:Cre mouse lines, respectively. We thank A. Chedotal, C. Goridis, and $G$. Rougon for critical reading of this manuscript. We also thank L. Richard and M. Piaser for their excellent technical assistance in electron microscopy.

F.C. and L.D. contributed equally to this work.

Correspondence should be addressed to Patrick Charnay, Developmental Biology Section, Inserm, Unité 1024, Centre National de la Recherche Scientifique, Unité Mixte de Recherche 8197, Institut de Biologie de l'Ecole Normale Supérieure, 46 rue d'Ulm, 75230 Paris Cedex 05, France. E-mail: patrick.charnay@ens.fr.

D0I:10.1523/JNEUROSCI.0017-10.2010

Copyright $\odot 2010$ the authors $\quad 0270-6474 / 10 / 305958-10 \$ 15.00 / 0$
}

spectively (Jessen and Mirsky, 2005; Richardson et al., 2006). During the embryonic period, the CNS/PNS interface is populated by another neural crest derivative, the boundary cap (BC) cells (Altman and Bayer, 1984).

We have shown previously that BC cells participate in the maintenance of the integrity of the CNS/PNS border in birds and mammals, because BC cell ablation results in the emigration of motoneuron cell bodies into the PNS (Vermeren et al., 2003). However, the mechanisms maintaining the segregation between central and peripheral glia are not known. Transgressions of the border by Schwann cells have been reported in rodent models with demyelinating spinal cord lesions and in some human neurological diseases, including multiple sclerosis (Itoyama et al., 1983; Duncan and Hoffman, 1997; Guest et al., 2005). Very recently, work performed in the zebrafish showed that Schwann cell depletion led to oligodendrocyte migration along the motor roots of embryos and larvae, suggesting that interaction between glial cells is important in this species (Kucenas et al., 2009).

In this study, to investigate the involvement of peripheral glia in the maintenance of the mammalian CNS/PNS boundary, we have taken advantage of different mouse mutations, in particular affecting the transcription factor gene Krox20 (Chavrier et al., 1988) (also known as Egr2), which is expressed in BC and Schwann cells and constitutes a master regulatory gene for myelination (Topilko et al., 1994; Svaren and Meijer, 2008). We demonstrate that ablation of Schwann and BC cells or inactivation of Krox20 results in an invasion of dorsal and ventral nerve 


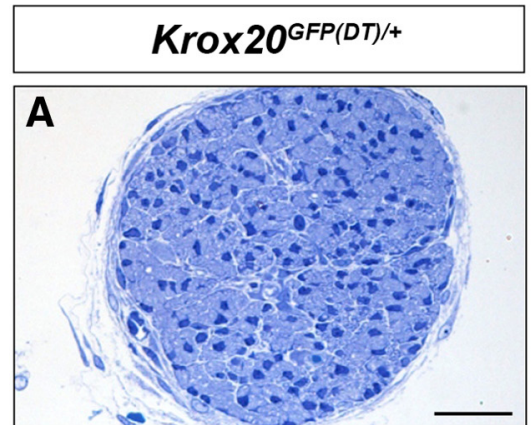

\section{HtPA:Cre,Krox $20^{\mathrm{GFP}(D T) /+}$}
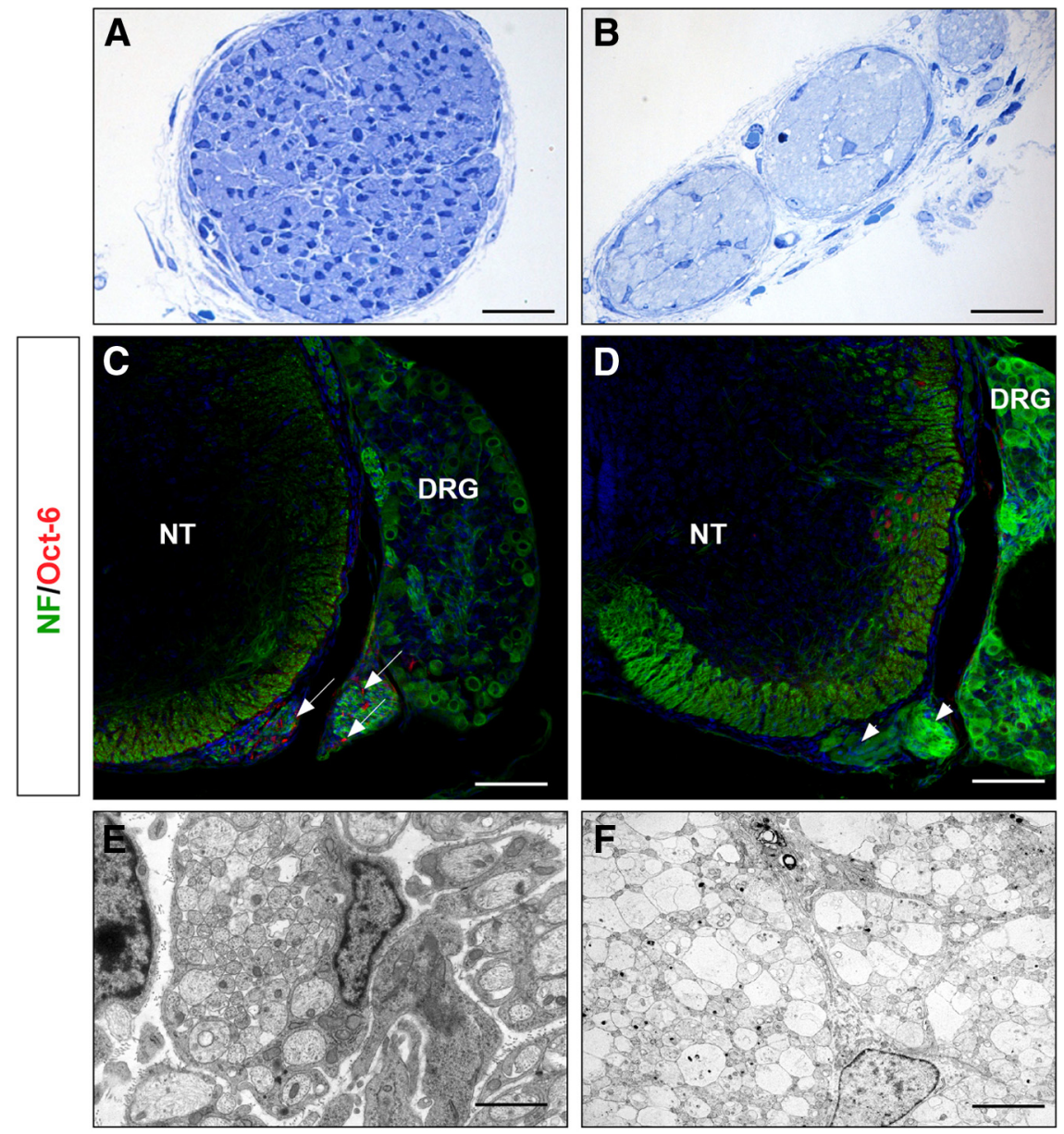

Figure 1. Absence of Schwann cells in the roots of $H t P A:\left(r e, K r o x 20^{G F P(D T) /+}\right.$ embryos. Histological $(\boldsymbol{A}, \boldsymbol{B})$, immunohistological $(\boldsymbol{C}, \boldsymbol{D})$, and ultrastructural $(\boldsymbol{E}, \boldsymbol{F})$ analyses of transverse sections through the dorsal nerve root from control (Krox $\left.20^{G F P(D T) /+}\right)$ and HtPA:Cre, Krox2 $0^{G F P(D T) /+}$ mutant E17.5 embryos. $A, B$, Note the reduction in size of the nerve and the presence of very few cells in the mutant. $\boldsymbol{C}, \boldsymbol{D}$, Immunohistochemistry for neurofilaments (NF) and the Schwann cell marker 0ct-6 shows the absence of Schwann cells in the mutant root. Arrows point to 0 ct-6-positive cells in the control $(\boldsymbol{C})$ and the arrowheads to the ventral nerve root in the mutant (D). The few 0ct-6-positive cells in the CNS are likely to correspond to neurons, as reported previously (Bermingham et al., 1996). $E$, $F$, Electron microscopy analysis of the nerve roots illustrates the different organization of the axons in the control and mutant embryos, with fascicles of directly adjacent axons in the mutant. NT, Neural tube. Scale bars: $A, B, 60 \mu \mathrm{m} ; \boldsymbol{C}, \boldsymbol{D}$, $120 \mu \mathrm{m} ; E, F, 6 \mu \mathrm{m}$.

roots by astrocytes and oligodendrocytes, followed by myelination of their axons by oligodendrocytes. This phenotype is not observed with the Trembler ${ }^{J}$ mutation, which blocks PNS myelination, but not Krox20 expression (Henry and Sidman, 1988; Giambonini-Brugnoli et al., 2005), suggesting that maintenance of the CNS/PNS boundary involves a Krox20 function that can be separated from myelination control. Furthermore, we also observed the presence of oligodendrocytes and astrocytes in the periphery in a human case of peripheral amyelinating neuropathy associated with absence of the KROX20 protein. Altogether, this work unravels important interactions between CNS and PNS glial cells, which may be critical in regenerative medicine.

\section{Materials and Methods}

Mouse lines and genotyping: ethical considerations. All mouse lines used in this study were maintained in a mixed C57BL/6/DBA2 background. We used the following alleles or transgenes that were genotyped as indicated in the original publications: $\operatorname{Krox} 20^{\text {Cre }}, \operatorname{Krox} 20^{G F P(D T)}, \operatorname{Krox} 20^{\text {flox }}$, Rosa26 $6^{\text {lac Z }}$, Rosa26 ${ }^{\text {YFP }}$, Trembler, HtPA:Cre, and Brn4:Cre (Suter et al., 1992;
Soriano, 1999; Voiculescu et al., 2000; Ahn et al., 2001; Srinivas et al., 2001; Taillebourg et al., 2002; Pietri et al., 2003; Vermeren et al., 2003). All animal manipulations were performed according to French and European Union regulations. Informed parental consents were obtained for the two patients (peripheral neuropathy and control), and the experiments were approved by the Ethical Committee of the Centre Hospitalier Universitaire Limoges.

Electron microscopy. Embryonic and postnatal spinal cord with attached dorsal root ganglia (DRGs) and nerve roots were fixed for $2 \mathrm{~h}$ in $1.6 \%$ glutaraldehyde in PBS and osmificated for $1 \mathrm{~h}$ in $1 \% \mathrm{OsO}_{4}$ (Polysciences). Nerve roots and sciatic nerve from patient were fixed for several days in $4 \%$ paraformaldehyde (PFA), then for $2 \mathrm{~h}$ in $1.6 \%$ glutaraldehyde in PBS, and osmificated for $1 \mathrm{~h}$ in $1 \% \mathrm{OsO}_{4}$. Samples were rinsed in PBS, dehydrated in graded acetone, and embedded in Epon. Semithin sections (1 $\mu \mathrm{m})$ were stained with toluidine blue. Ultrathin sections were stained with uranyl acetate and lead citrate. The sections were observed through a JEOL electron microscope. $g$-ratios were estimated from $>100$ myelinated axons per mouse and three mice per genotype.

Immunohistochemistry and detection of $\beta$-galactosidase activity. For immunohistochemistry, two embedding protocols were used depending on the antibody used for labeling. In the first case, embryonic and postnatal spinal cord with DRGs and nerve roots were collected, fixed in $4 \%$ PFA for $2 \mathrm{~h}$, incubated overnight at $4^{\circ} \mathrm{C}$ in $15 \%$ sucrose, embedded in Tissue-Tek OCT compound (Gassalem), and frozen on dry ice. In the second case, samples were fixed in 4\% PFA for $2 \mathrm{~h}$, rinsed in PBS buffer, dehydrated in graded ethanol, and embedded in Polyesther Wax (BDH). Spinal cord and nerve root sections were prepared from the thoracic level unless otherwise stated. Transverse sections ( $12 \mu \mathrm{m}$ thick) were collected using a Leica cryotome. Sections were incubated for $30 \mathrm{~min}$ in $0.1 \mathrm{~m}$ PBS containing $5 \%$ normal goat serum and $0.25 \%$ Triton X-100 and then overnight at $4^{\circ} \mathrm{C}$ with the following primary antibodies: rabbit anti-green fluorescent protein (GFP) (1:500; Invitrogen), rabbit antiOlig2 (1:400; Millipore Bioscience Research Reagents), mouse monoclonal anti-P0 (1:5; obtained from Dr. Thomas, Paris, France), mouse monoclonal anti-2H3/neurofilament (1:1000; Developmental Studies Hybridoma Bank, Universal of Iowa, Iowa City, IA), mouse monoclonal anti-myelin oligodendrocyte glycoprotein (MOG) (1:100; ascites obtained from Dr. Linnington, Planegg-Martinsried, Germany), mouse monoclonal anti-glial fibrillary acidic protein (GFAP) (1:100; Millipore Bioscience Research Reagents), mouse monoclonal anti-PAN$\mathrm{Na}_{\mathrm{v}}$ (1:100; Sigma), and rabbit anti-Paranodin (1:500; obtained from Dr. Goutebroze, Paris, France). Sections were then washed and incubated for $150 \mathrm{~min}$ at room temperature with the appropriate fluorophoreconjugated secondary antibody (Jackson ImmunoResearch). Nuclei were counterstained with Hoechst (33342; Sigma). Finally, sections were mounted in Aqua poly/Mount (Polysciences) and analyzed on Leica DMRD and TCS 4D confocal microscopes. Whole-mount $\beta$-galactosidase detection was performed as described previously (Sham et al., 1993). For human samples, spinal cords and nerve roots were fixed in $4 \%$ PFA. Transverse sections ( $5 \mu \mathrm{m}$ thick) were collected using a Leica microtome. Sections were incubated overnight at $4^{\circ} \mathrm{C}$ with the following primary antibodies: rabbit anti-GFAP (1:500; Dako), mouse monoclonal 


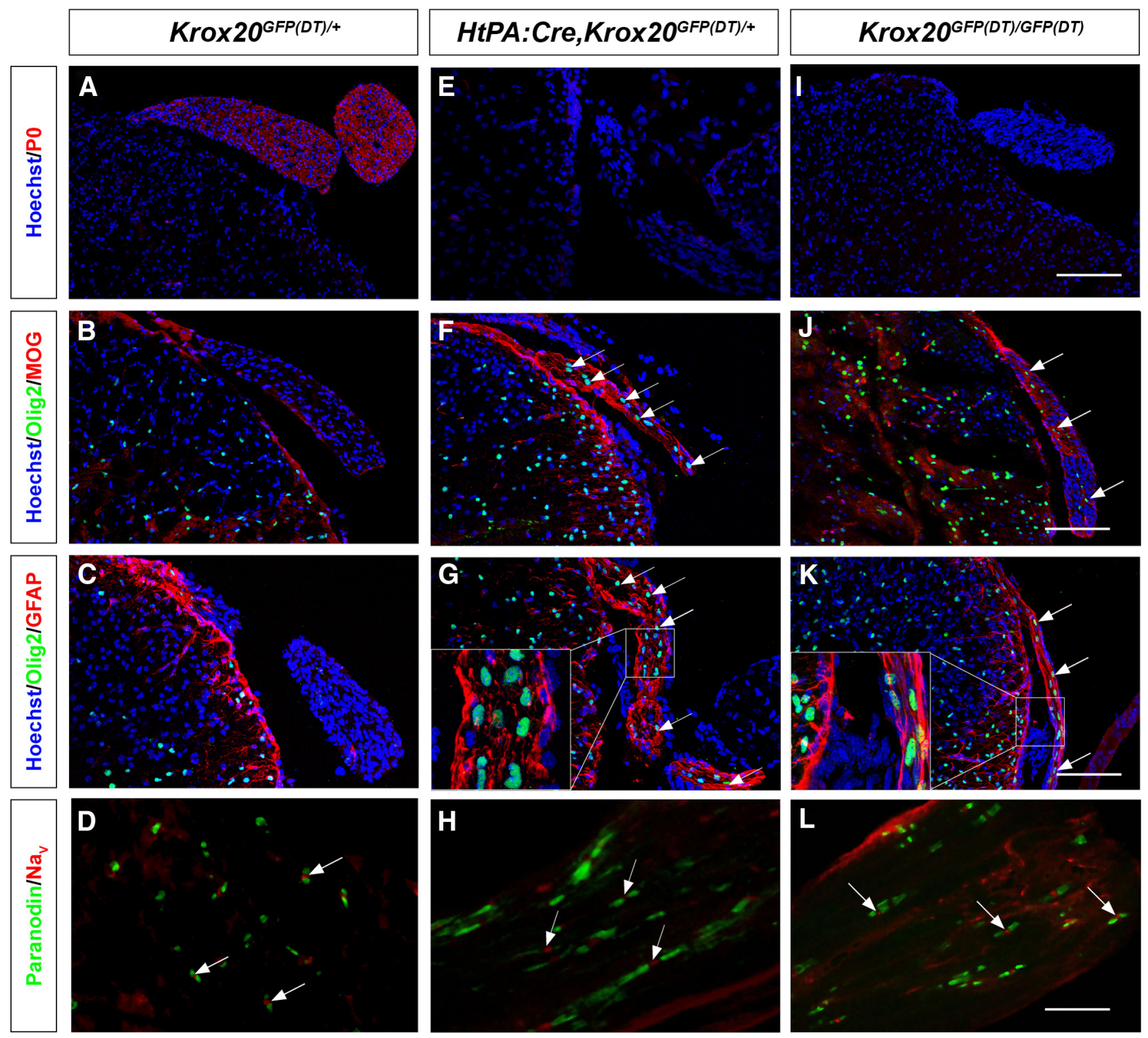

Figure 2. Presence of CNS-type glia in the nerve roots from Schwann cell-depleted and Krox20 null mice. Transverse sections through the spinal cord and dorsal nerve root from P11-P14 control $\left(\right.$ Krox $\left.20^{G F P(D T) /+}\right)$, Schwann cell-depleted (HtPA:Cre,Krox $\left.20^{G F P(D T) /+}\right)$, and Krox20 null (Krox2 $0^{G F P(D T) / G F P(D T)}$ ) mice were analyzed by immunohistochemistry for various (NS and PNS markers as indicated, and the nuclei were counterstained with Hoechst $33342 . A, E, I$, Peripheral myelin marker P0 analysis shows that the mutant nerve roots are devoid of peripheral myelin. $B, F, J, T h e$ mutant nerve roots contain central myelin as indicated by the labeling for the central myelin component MOG. The arrows point to nuclei positive for 0 lig 2 in the roots. $C, G, K$, The mutant nerve roots contain oligodendrocytes and astrocytes, as revealed by the 0lig2 and GFAP markers, respectively. The arrows point to nuclei positive for 0lig 2 in the roots. $D, H, L$, The nodal/paranodal organization is preserved in the mutant roots myelinated by oligodendrocytes. The arrows point to nodes of Ranvier showing $\mathrm{Na}_{\mathrm{v}}$ and paranodin expression. The insets in $\boldsymbol{G}$ and $\boldsymbol{K}$ correspond to higher magnification of the indicated areas. Scale bars: $\boldsymbol{A}-\boldsymbol{C}, \boldsymbol{E}-\mathbf{G}, \mathbf{I}-\boldsymbol{K}, 120 \mu \mathrm{m} ; \mathbf{D}, \boldsymbol{H}, \mathbf{L}, 14 \mu \mathrm{m}$.

anti-proteolipid protein (PLP) (1:400; Serotec), and rabbit anti-EGR2 (1:400; Aviva Systems). Sections were then washed with Tris buffer, $\mathrm{pH}$ 7.4. The LSAB + kit (Dako) was used to reveal the presence of primary antibodies. Nuclei were counterstained with hematoxylin.

\section{Results}

Generation of a mouse mutant depleted of Schwann and BC cells

To investigate the contribution of peripheral glia to the CNS/PNS boundary, we performed their genetic ablation in the mouse. For this purpose, we took advantage of a knock-in allele, $\operatorname{Krox} 20^{G F P(D T)}$, in which the Krox20 coding sequence has been replaced by a cassette containing the GFP coding sequence, flanked by loxP sites and followed by the coding sequence for the
A chain of the diphtheria toxin (Vermeren et al., 2003). In this configuration, when the Krox20 locus is activated, only the GFP gene is expressed. In contrast, after Cre-mediated recombination, activation of the locus leads to expression of the diphtheria toxin gene and subsequent cell death. Schwann and BC cells are the only known neural crest derivatives expressing Krox20, apart from a subpopulation derived from rhombomere 5 (Ghislain et al., 2003). Therefore, by combining the $\operatorname{Krox} 20^{G F P /(D T)}$ allele with a Cre driver whose expression is restricted to the neural crest [HtPA:Cre (Pietri et al., 2003)], it should be possible to obtain an almost specific ablation of Schwann and BC cells and to avoid the embryonic lethality attached to the elimination of all Krox20expressing cell types (Vermeren et al., 2003). Breeding of HtPA:Cre 

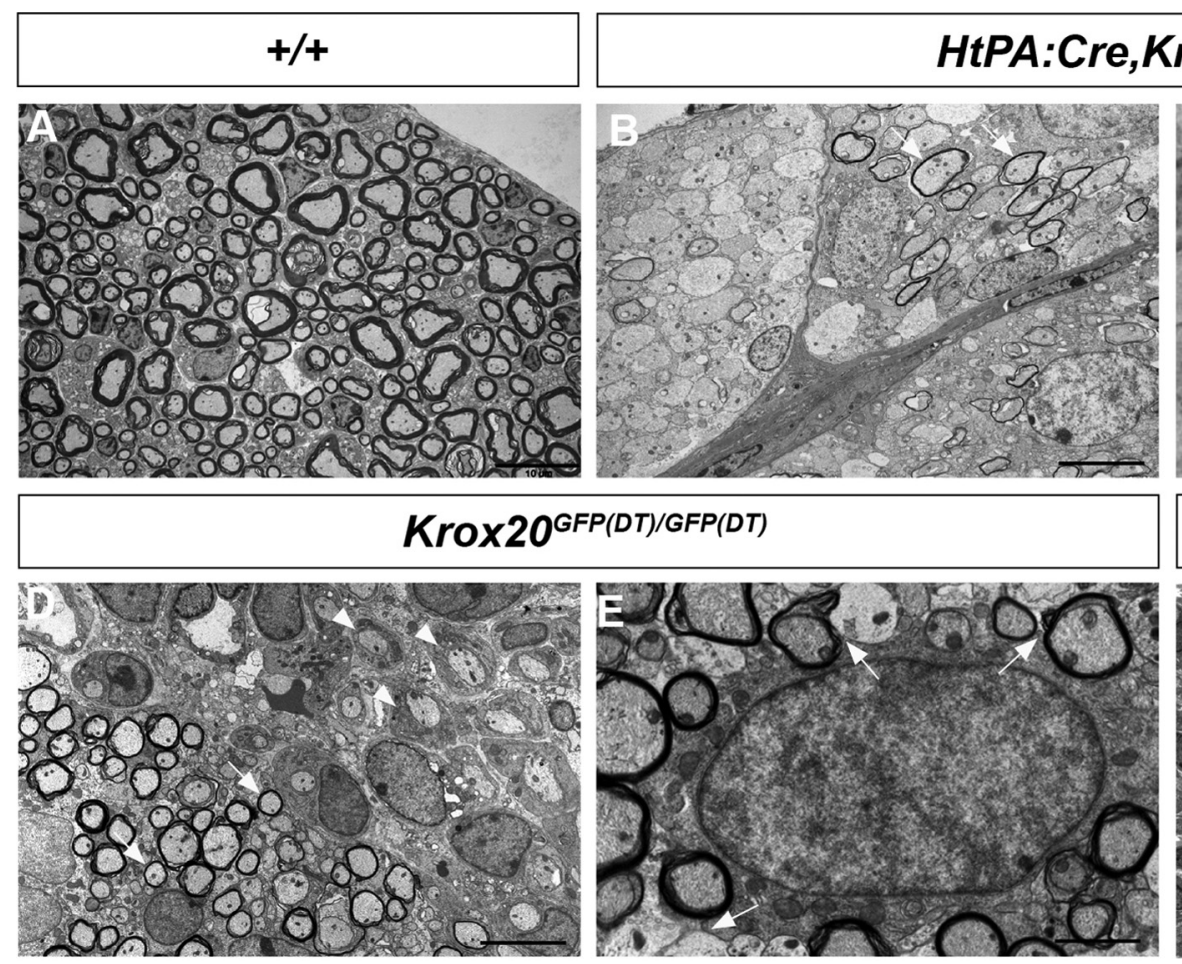

\section{FP(DT)/GFP(DT)}
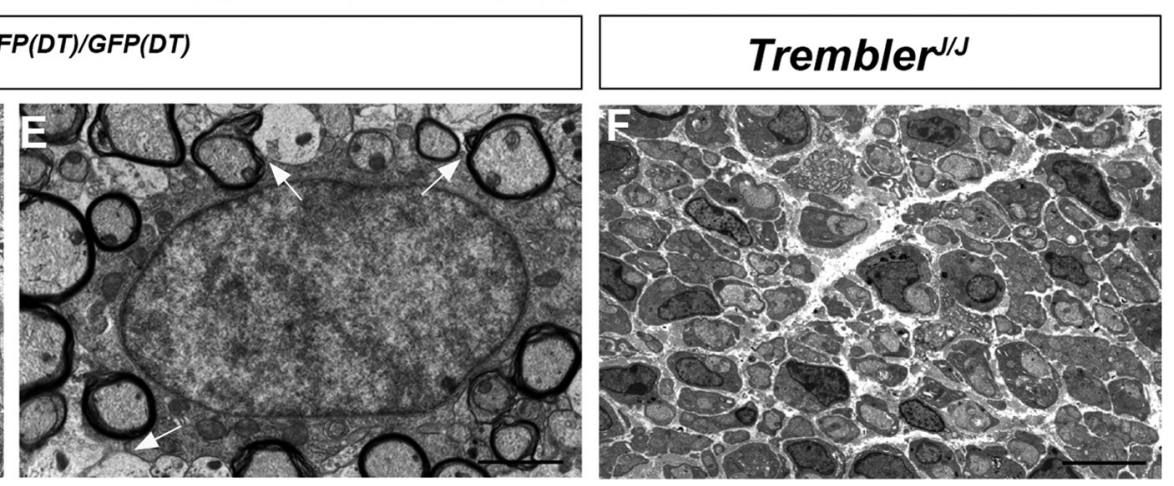

Figure 3. Electron microscopy analysis of the nerve roots from mutant mice. Analysis of transverse sections through P14 wild-type $(\boldsymbol{A},+/+)$, Schwann cell-depleted $(\boldsymbol{B}, \boldsymbol{C}, H t P A$ : Cre,Krox $\left.20^{G F P(D T) /+}\right)$, Krox20 null $\left(\boldsymbol{D}, \boldsymbol{E}, \operatorname{Krox} 20^{G F P(D T) / G F P(D T)}\right)$, and Trembler ${ }^{/ / J}(\boldsymbol{F})$ nerve roots reveals the presence of thin myelin sheaths (arrows), presumably generated by oligodendrocytes, in the Schwann cell-depleted ( $\boldsymbol{B})$ and Krox20 null (D) mutants and confirms the absence of myelin in the Trembler//J mutant $(\boldsymbol{F})$. In the case of the Krox20 null mutant, the oligodendrocytes (D, arrows) coexist with Schwann cells blocked at the promyelinating stage ( $\boldsymbol{D}$, arrowheads). High magnification allows the observation of astrocyte-specific gliofilaments ( $\boldsymbol{C}$, arrow) and of a thin rim of homogeneous cytoplasm without fibrils around the nucleus, characteristic of oligodendrocytes $(\boldsymbol{E})$ and cellular processes ensheathing different axons $(\boldsymbol{E}$, arrows). Scale bars: $\boldsymbol{A}, \boldsymbol{B}, \boldsymbol{D}, \boldsymbol{F}, 10 \mu \mathrm{m} ; \boldsymbol{C}, \boldsymbol{D}, 2 \mu \mathrm{m}$.

and $\operatorname{Krox} 20^{G F P(D T) /+}$ animals gave rise to HtPA:Cre,Krox $20^{G F P(D T) /+}$ newborns in a normal Mendelian ratio (data not shown), and these animals survived until $\sim 3$ weeks after birth. The ablation should occur after the activation of Krox20 in each cell type, i.e., at approximately embryonic day 10.5 (E10.5) for BC cells and E15.5 for Schwann cells. We first performed analyses of the nerve roots from the mutant animals at E17.5 $(n=3)$, which revealed a severe reduction of the thickness of the mutant nerve compared with the control (Fig. $1 A, B$ ). The number of cells was dramatically reduced, with no indication of the presence of Schwann cells, as illustrated by the absence of the Oct-6-positive cells (Fig. 1C,D). Electron microscopy analysis showed that, in the mutant, the axons were directly adjacent and organized in fascicles, whereas in the control, the axons were surrounded by cytoplasmic extensions from the Schwann cells (Fig. $1 E, F$ ). The very severe reduction or absence of Schwann cells was also supported by the lack of peripheral myelin at later stages (Fig. 2A,E). Efficient recombination in $\mathrm{BC}$ cells and their elimination in the mutants were established by the absence of GFP expression (supplemental Fig. $1 A, B$, available at www.jneurosci.org as supplemental material) and Krox20 and L20 mRNAs (data not shown) [L20 is another BC cell marker (Coulpier et al., 2009)] at the level of nerve entry and exit points at E12.5. As expected from previous work (Vermeren et al., 2003), elimination of BC cells resulted in the emigration of some motoneuron cell bodies along the ventral nerve root at E12.5 (supplemental Fig. $2 A, B$, available at www.jneurosci.org as supplemental material). Altogether, these data indicate that, as expected, the HtPA:Cre,Krox $20^{G F P(D T) /+}$ mutants are devoid of Schwann and $\mathrm{BC}$ cells, and, for convenience, we will now refer to these animals as Schwann cell-depleted.
Oligodendrocytes, astrocytes, and myelin are present in the nerve roots of Schwann cell-depleted animals

We performed a detailed immunohistochemistry analysis of spinal nerve roots at thoracic level from HtPA:Cre, Krox $20^{G F P(D T) /+}$ mutants and showed that, at postnatal day 11 (P11) to P14, these animals $(n=4)$ were devoid of peripheral myelin, as indicated by the absence of P0 protein (Fig. 2A,E). Surprisingly, the nerve roots were positive for two central myelin markers, MOG and PLP (Fig. $2 B, F$ and data not shown). The roots were also positive for GFAP, specific to astrocytes and for the Olig2 nuclear factor, which constitutes a marker of oligodendrocytes and their precursors (Fig. $2 B, C, F, G$ ). Olig2 and MOG were also detected in cranial nerve roots (supplemental Fig. 3, available at www.jneurosci. org as supplemental material). These data suggest that CNS-type myelin is present in the peripheral roots and that the ectopic oligodendrocytes are responsible for its synthesis. In addition, we always observed a colocalization of ectopic oligodendrocytes and astrocytes in the nerve roots (Fig. 2C,G) but never found either type of central glia in distal parts of the nerve (data not shown).

The presence of astrocytes and myelinating oligodendrocytes in Schwann cell-depleted animals was further supported by electron microscopy analysis of the nerve roots from P14 animals $(n=3)$ (Fig. $3 A-C)$. Ectopic astrocytes were identified by the presence of the gliofilaments of specific thickness $(9.76 \pm 2.15$ $\mathrm{nm}$, mean \pm SEM) (Fig. $3 C$, arrows). The identity of oligodendrocytes was supported by the analysis of the $g$-ratios (ratio between the axon diameter and total fiber diameter). In wild-type mice, in which the axons are ensheathed by Schwann cells (Fig. $3 A$ ), the $g$-ratio was $0.67 \pm 0.04$, in agreement with published data (Friede, 1986). In contrast, in the mutant nerve roots, the 
myelin was significantly thinner ( $g$-ratio of $0.83 \pm 0.04$ ) (Fig. 3B). No significant difference was found between the $g$-ratios of wild-type and mutant animals within the spinal cord $(0.78 \pm 0.04$ vs $0.79 \pm$ $0.03)$. In the mutants, the $g$-value of root myelin is therefore closer to the $g$-ratio of central myelin, consistent with the view that root myelin is generated by oligodendrocytes.

Finally, immunostaining for $\mathrm{Na}_{\mathrm{v}}$, the sodium channels present at the mature nodes of Ranvier and for paranodin, a marker of the paranode, revealed an apparently normal organization of the node/paranode structure of the oligodendrocyte-myelinated axons of the nerve roots (Fig. $2 \mathrm{D}, \mathrm{H}$ ).

\section{Nerve roots of Krox 20 null mice also contain central-type myelin}

The presence of central-type myelin in the nerve roots of Schwann cell-depleted animals might be attributable to the absence of Schwann cells or simply of peripheral myelin. To distinguish between these two possibilities, we analyzed Krox20 null mutants $\left(\operatorname{Krox} 20^{G F P(D T) / G F P(D T)}\right)$, in which Schwann cells are present but unable to synthesize myelin (Fig. 3D). Immunohistochemistry analyses revealed that, like Schwann cell-depleted animals, spinal nerve roots from P14 Krox20 null mutants $(n=4)$ were negative for the peripheral myelin marker $\mathrm{P} 0$ and positive for MOG, PLP, Olig2, and GFAP (Fig. $2 \mathrm{I}-\mathrm{K}$ and data not shown). The presence of myelinating oligodendrocytes and astrocytes was further supported by electron microscopy analyses (Fig. 3D,E). Identification of oligodendrocytes was based on the estimation of $g$-ratios $(0.80 \pm 0.03)$ and on the presence of a thin characteristic rim of homogeneous cytoplasm without fibrils around the nucleus (Peters et al., 1991) (Fig. 3E). Furthermore, several myelinated axons were closely applied to the cytoplasm of some cells whose cellular processes ensheath these axons (Fig. 3E, arrows). These data suggest that a cell can myelinate several axons, a characteristic that differentiates oligodendrocytes from Schwann cells. In addition, this analysis revealed the coexistence of the oligodendrocytes with Schwann cells blocked at the promyelinating stage (Fig. $3 D)$. In all examined animals $(n=3)$, the oligodendrocytes and astrocytes occupied the center of the root, whereas the Schwann cells were present at the periphery (data not shown). Immunostaining for $\mathrm{Na}_{\mathrm{v}}$ and paranodin confirmed that the myelin generated by the ectopic oligodendrocytes presented an apparently normal organization of the node/paranode structure (Fig. $2 L$ ). In distal parts of the peripheral nerves, only Schwann cells were observed and GFAP- and Olig2-positive cells were not detected, indicating that ectopic astrocytes and oligodendrocytes are restricted to the nerve roots (data not shown).

The Krox20 null mice usually die within 2 weeks after birth. To investigate whether the presence of central glial components in the spinal roots is a permanent feature, we made use of a conditional mutant, Krox $20^{\text {Crefflox }}$, that combines a floxed Krox 20 allele with a knock-in of the Cre recombinase in the Krox20 locus (Voiculescu et al., 2000; Taillebourg et al., 2002). In this mutant, the Krox20 gene is expressed only very transiently. This does not prevent the block in peripheral myelin formation but alleviates other phenotypes and allows a much longer survival of the animals (Decker et al., 2006). Immunohistological analysis of the dorsal and ventral nerve roots from P42 Krox $20^{\text {Crefflox }}$ animals $(n=3)$ established the absence of P0 (supplemental Fig. 4A, $B$, available at www.jneurosci.org as supplemental material) and revealed the presence of centraltype myelin, oligodendrocytes, and astrocytes (supplemental Fig. $4 C-H$, available at www.jneurosci.org as supplemental material). Therefore, the presence of ectopic oligodendrocytes appears to be a permanent feature of Krox20-deficient animals. Altogether, our data indicate that the absence of Schwann cells is not necessary for the presence of central-type glia in the nerve roots and that the mutant nerve root environment is permissive for their long-term survival.

\section{Absence of central-type glia in Trembler ${ }^{\mathrm{J} / \mathrm{I}}$ mice}

The above data established that inactivation of Krox20 is sufficient for the presence of central-type myelin in the nerve roots. Krox20 inactivation leads to the complete absence of peripheral myelin and affects the expression of a number of genes, including myelin genes (Topilko et al., 1994). The presence of CNS-type 

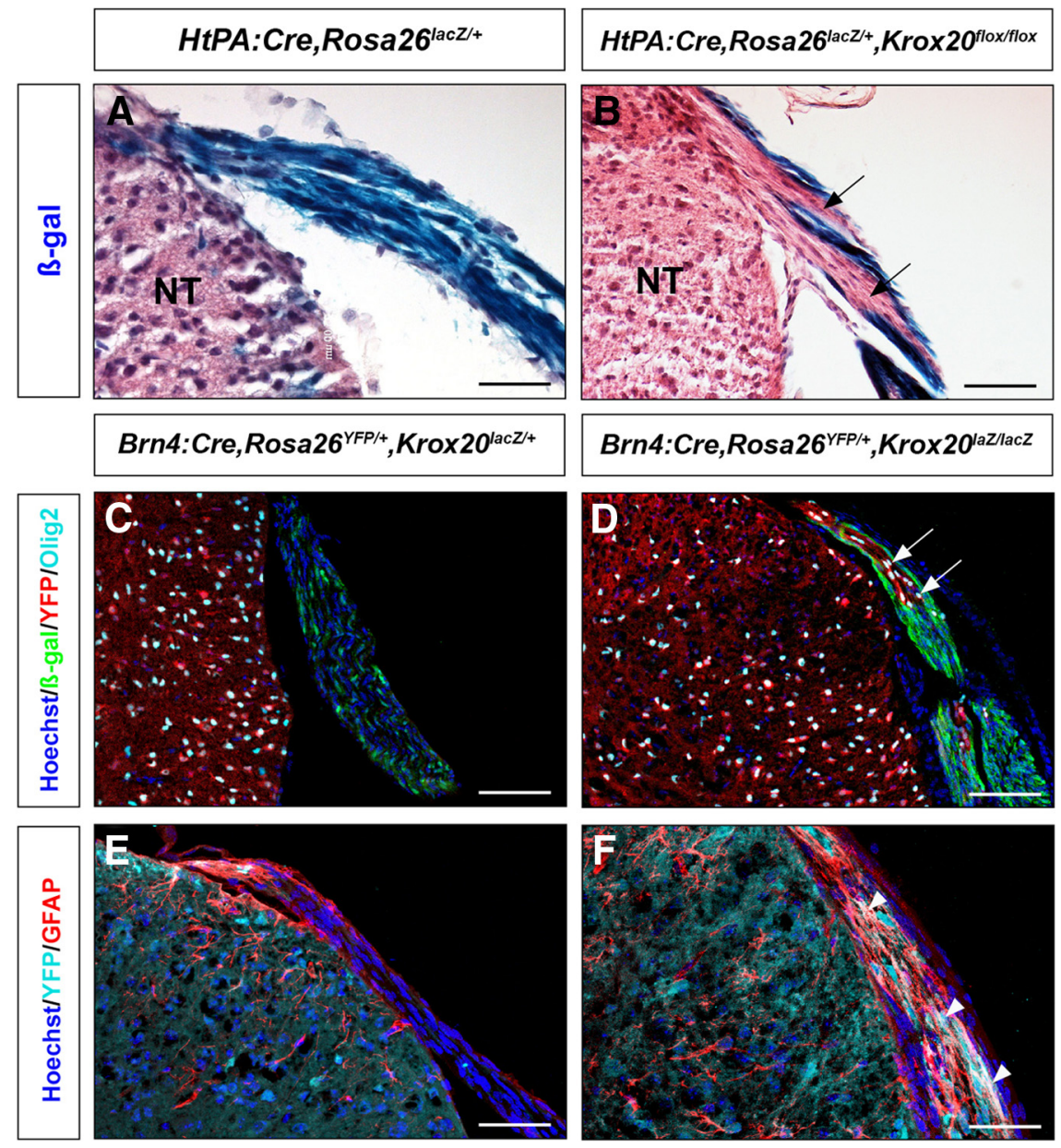

Figure 5. Origin of the ectopic oligodendrocytes and astrocytes of the nerve roots. $A, B$, Genetic tracing of neural crest derivatives in the dorsal nerve roots of P14 control $(\boldsymbol{A})$ and Krox20 null mutant $(\boldsymbol{B})$ mice. lacZ is expressed in neural crest derivatives and labels essentially all the cells in the roots of the control animal $(\boldsymbol{A})$ and only some of them in the Krox20 null mutant ( $\boldsymbol{B}$; arrows point to regions devoid of $\beta$-galactosidase labeling). $\boldsymbol{C}-\boldsymbol{F}$, Genetic tracing of CNS derivatives in the dorsal nerve roots of P14 control (C, $\boldsymbol{E})$ and Krox20 null mutant ( $\boldsymbol{D}$, $\boldsymbol{F}$ ) mice. No CNS derivatives (YFP positive, red in $\boldsymbol{C}, \boldsymbol{D}$; light blue in $\boldsymbol{E}, \boldsymbol{F}$ ) are observed in the roots of the control animals, whereas many are found in the mutants. All Olig2-positive nuclei ( $\boldsymbol{D}$, arrows) and GFAP-positive cells ( $\boldsymbol{F}$, arrowheads) in the mutant dorsal roots correspond to YFP-positive cells, therefore derived from the CNS. NT, Neural tube. Scale bars: $\boldsymbol{A}-\boldsymbol{D}, 120 \mu \mathrm{m} ; \boldsymbol{E}, \boldsymbol{F}, 75 \mu \mathrm{m}$.

glial components in the roots of Krox20 null mice might therefore be a consequence of the absence of peripheral myelin or a defect in another Krox20 function. To differentiate between these two possibilities, we made use of another mutant, Trembler ${ }^{J / J}$, which carries a missense mutation in the PMP22 coding sequence (Sidman et al., 1979). PMP22 is a transmembrane protein involved in peripheral myelin compaction, and this mutation completely prevents the formation of myelin (Henry and Sidman, 1988), as shown by electron microscopy (Fig. $3 F$ ) and the absence of $\mathrm{P} 0$ expression (Fig. 4A). Interestingly, Krox20 is normally expressed in this mutant (Giambonini-Brugnoli et al., 2005) (Fig. 4B). Immunohistochemical analysis of nerve roots from P14 mutant animals $(n=$ 3) failed to detect cells positive for Olig2 or GFAP (Fig. 4C). The central myelin protein MOG was also not found in the nerve roots (Fig. 4D). These observations indicate that the lack of peripheral myelin is not sufficient for the presence of myelinating oligodendrocytes and astrocytes in the nerve roots. They suggest that this is a defect in another Krox20 function that is responsible for this phenotype.

\section{Ectopic central glia are derived from the CNS by} progressive migration

The ectopic astrocytes and oligodendrocytes observed in the nerve roots of the Schwann cell-depleted and Krox20 null mu- tants might derive from PNS cells by transdifferentiation or CNS cells by migration. Transgression of the CNS/PNS border by oligodendrocytes has never been reported in mammals, but the potential of the neural crest-derived glial satellite cells to adopt oligodendrocyte characteristics in vitro has been described recently (Svenningsen et al., 2004). As indicated above, we never detected CNStype glial components in distal parts of peripheral nerves from both mutants, an observation consistent with the migration hypothesis. To definitively establish the origin of the oligodendrocytes and astrocytes in the mutant nerve roots, we performed lineage tracing experiments. Two Cre-driver lines, HtPA:Cre and Brn4:Cre, the latter being active in the entire neural tube as early as E9.5 (Ahn et al., 2001), were used in combination with Rosa $26^{\text {lac } Z}$ and Rosa $26^{Y F P}$ alleles, which lead to permanent activation of the reporter gene during Cre recombination (Srinivas et al., 2001; Ille et al., 2007), to label neural crest and CNS derivatives, respectively. These combinations were transferred into Krox20 null mutant or control backgrounds, and reporter expression was analyzed in the nerve roots of $\mathrm{P} 14$ animals in combination with the Olig2 and GFAP markers. Analysis of HtPA ${ }^{\text {Cre }}$, Rosa $26^{\text {lacz/ }}$ ,+ Krox $20^{\text {flox/flox }}$ animals showed that their nerve roots were heterogeneous, containing cells positive and negative for the $\beta$-galactosidase marker, in contrast to the control situation in which most of the cells were $\beta$-galactosidase positive (Fig. $5 A, B)$. These data suggested that the mutant nerve roots were in part populated by cells that were not of neural crest origin. Analysis of Brn4:Cre, Rosa26 $6^{\text {YFP/+ }}$, Krox $20^{\text {lacZ/lacZ }}$ animals showed that yellow fluorescent protein (YFP)-positive cells were found in the nerve roots and that all cells expressing Olig2 or GFAP were also YFP positive (Fig. $5 D, F$ ), in contrast to the control situation in which the nerve roots were totally devoid of YFP-, Olig2-, or GFAP-positive cells (Fig. 5C,E). Altogether, these data indicate that Krox20 null mutant nerve roots are in part populated by cells that derive from the CNS and not from the neural crest and that are likely to have migrated along the roots. The ectopic oligodendrocytes and astrocytes belong to this immigrant population and must therefore derive from Brn4-positive precursors rather than from transdifferentiated peripheral cells.

Because the central glial components of the roots originate from the CNS, it should be possible to observe a progressive colonization. We therefore performed a time course analysis of the presence of oligodendrocytes between E15.5 and P14 in both HtPA:Cre, Krox $20^{G F P(D T) /+}$ and $\operatorname{Krox} 20^{G F P(D T) / G F P(D T)}$ nerve roots. In the Schwann cell-depleted animals, ectopic oligodendrocytes were observed in the roots as early as E17.5 (Fig. 6A, $B$ ) and thereafter (Fig. 6D). In contrast, in the Krox20 null mutant, no ectopic oligodendrocytes were found at E17.5 or P1-P2 (Fig. $6 C$ and data not shown), and they were only detected from P3 
(Fig. 6D). These data indicate that colonization of the nerve roots by CNS glia occurs earlier in the Schwann cell-depleted embryos than in Krox20 null mutants. Furthermore, evaluation of the number of ectopic oligodendrocytes indicated that colonization of the roots was also more efficient in Schwann cell-depleted embryos at all analyzed stages (Fig. 6D). Finally, the fact that, in the latter embryos, the migration is observed from E17.5 at least, i.e., before the initiation of peripheral myelin formation, is consistent with the absence of link between this migration and the lack of myelin.

\section{CNS/PNS boundary transgression in a human patient}

In the search for human cases that might present a nerve root phenotype similar to those observed in mice, we investigated a patient affected by a very severe congenital peripheral neuropathy, who died at 7 months. Ultrastructure analysis of sciatic nerve sections revealed a situation very similar to that observed in Krox20 null mouse mutants, with a complete absence of myelin and Schwann cells apparently blocked at the promyelinating stage (Fig. $7 A, B)$. Consistently, the KROX20 protein was not detected in the nuclei of the patient's Schwann cells (Fig. 7C,D). There were no histological lesions of the CNS white matter (data not shown). Immunohistochemical analysis of the nerve roots revealed the presence of cells positive for GFAP, Olig2, or PLP, in contrast to a control individual (Fig. 8), therefore suggesting that the roots contained astrocytes, oligodendrocytes, and central myelin. These data suggest that transgression of the CNS/PNS boundary can also occur in humans and may originate from the same defects as in mice.

\section{Discussion}

In previous work, we have shown that $\mathrm{BC}$ cells are involved in maintaining the CNS/ PNS boundary in mouse and chick because their ablation leads to the emigration of some of the motoneurons into the periphery (Vermeren et al., 2003). The present study presents the first examples in mammals of transgressions of the boundary in which glial components of the CNS invade the PNS. We have shown that, in different mouse mutants and in a human case presenting defects in Schwann cells and BC cells, oligodendrocytes and astrocytes colonize the dorsal and ventral nerve roots, leading to the formation of bona fide central myelin in the periphery. Our observations provide some clues to the mechanisms governing the maintenance of the CNS/PNS boundary and raise interesting issues concerning cell transplantation and applications to human pathology.

D
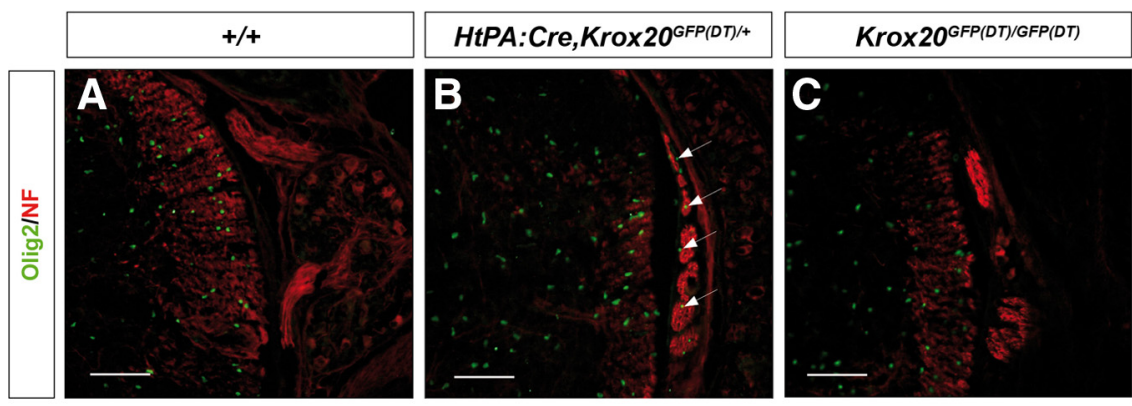

\begin{tabular}{|c|c|c|c|c|}
\hline 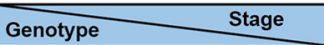 & E15.5 & E17.5 & P3 ${ }^{*}$ & P14 ${ }^{* *}$ \\
\hline HtPA:Cre,Krox $20^{G F P(D T) /+}$ & 0 & $4.3+/-0.88$ & $4.25+/-0.98$ & $6+/-0.70$ \\
\hline Krox $20^{-1-}$ & 0 & 0 & $1.8+/-0.48$ & $3.9+/-0.49$ \\
\hline
\end{tabular}

Figure 6. Differences in time courses of central glia emigration between mouse mutants. Transverse sections through the spina cord and dorsal nerve root from E17.5 wild-type $(\boldsymbol{A},+/+)$, Schwann cell-depleted $\left(\boldsymbol{B}, H t P A:\left(r e, K r o \times 20^{G F P(D T) /+}\right)\right.$, and Krox20 null (C, Krox20 $0^{G F(D T) / G F P(D T)}$ ) embryos were analyzed by immunohistochemistry for neurofilaments (NF) and the oligodendrocyte marker Olig2. The arrows in $\boldsymbol{B}$ point to 0 lig2-positive nuclei in the nerve root. Scale bars, $120 \mu \mathrm{m}$. The table in $\boldsymbol{D}$ shows the mean \pm SEM number of 0lig2-positive nuclei found per 100- $\mu \mathrm{m}$-long segment, on $12-\mu \mathrm{m}$-thick dorsal root longitudinal sections in

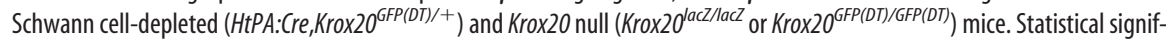
icance was analyzed using the Student's $t$ test; ${ }^{*} p<0.03 ;{ }^{* *} p<0.02$.
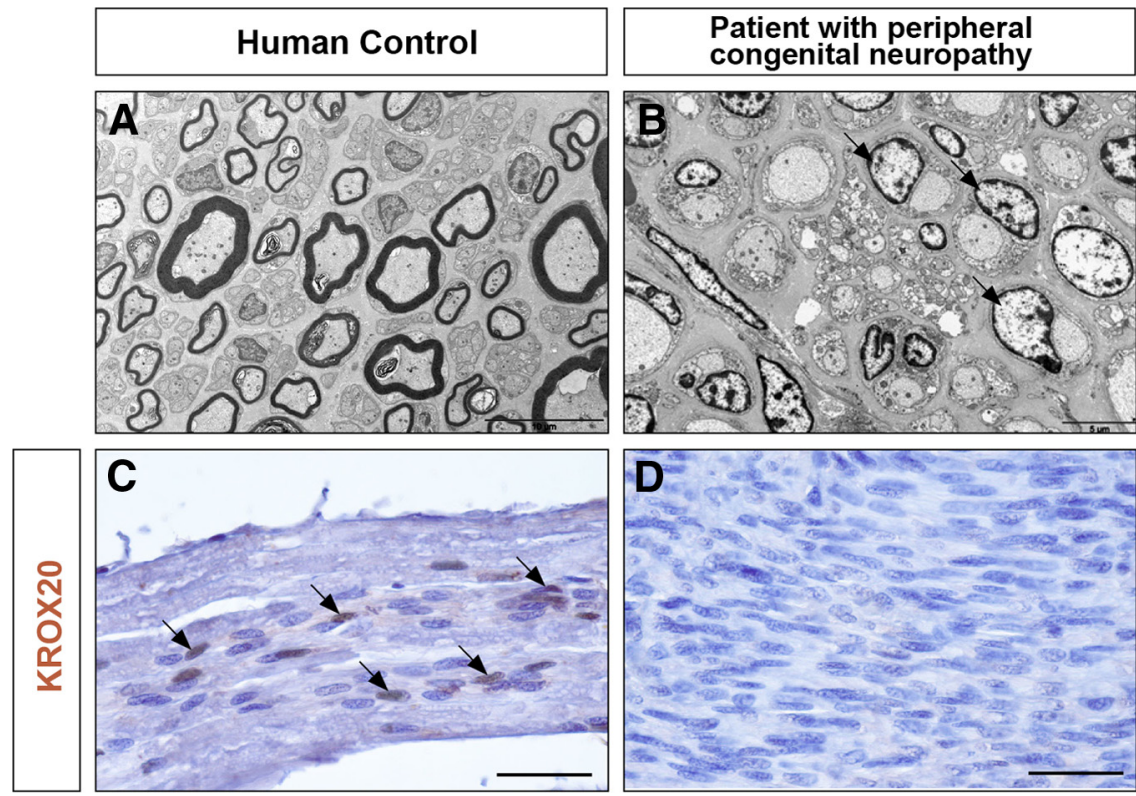

Figure 7. Absence of myelin and of KROX20 protein in the sciatic nerve of a patient with congenital peripheral neuropathy. Transverse sections through the sciatic nerves from a 12-month-old control $(\boldsymbol{A})$ and the 7-month-old peripheral neuropathy patient $(\boldsymbol{B})$ were analyzed by electron microscopy. Longitudinal sections through the dorsal root from the same control $(\boldsymbol{C})$ and the peripheral neuropathy patient $(\boldsymbol{D})$ were analyzed by immunohistochemistry for KROX20. Note the absence of myelin and the presence of Schwann cells apparently blocked at the promyelinating stage ( $\boldsymbol{B}$, arrows) in the patient's nerve. In $\boldsymbol{C}$, arrow point to KROX20-positive nuclei, which are absent in $\boldsymbol{D}$. Scale bars: $\boldsymbol{A}, 10 \mu \mathrm{m} ; \boldsymbol{B}, 5 \mu \mathrm{m} ; \boldsymbol{C}, \boldsymbol{D}, 40 \mu \mathrm{m}$.

\section{Control of CNS/PNS boundary}

Using morphological and immunological characterizations, time course analyses, and genetic tracing, we have shown that massive invasion of the nerve roots by oligodendrocytes and astrocytes occurs, presumably at all axial levels, in mice in which Schwann and BC cells have been genetically ablated or Krox20, a master gene for PNS myelination, has been inactivated. The fact that this invasion also occurs in the Krox20 mutant, in which Schwann cells are still present, indicates that there is indeed a transgression of the boundary and not simply a displacement. The ectopic oli- 


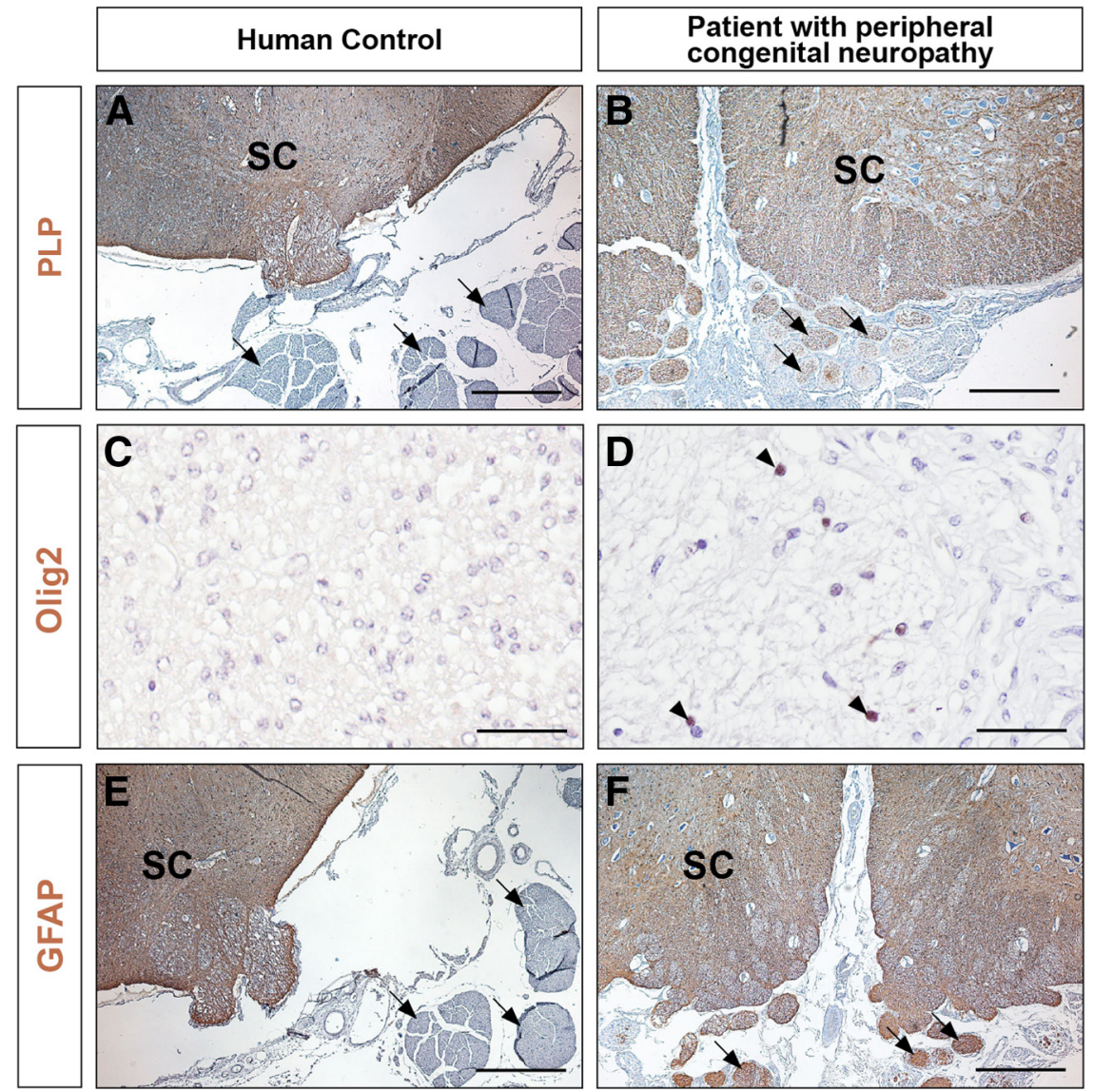

Figure 8. Presence of CNS-type glia in the nerve roots from a patient with congenital peripheral neuropathy. Transverse sections through the spinal cord and nerve roots from a 12 -month-old control $(A, C, E)$ and the 7-month-old peripheral neuropathy patient $(\boldsymbol{B}, \boldsymbol{D}, \boldsymbol{F})$ were analyzed by immunohistochemistry for the presence of PLP, 0lig2, and GFAP, markers of central myelin, oligodendrocytes, and astrocytes, respectively. In $C, D$, the magnification restricts the field to the nerve root. The arrows point to nerve roots and the arrowheads to Olig2-positive cells. SC, Spinal cord. Scale bars: $A, B, E, F, 250 \mu \mathrm{m} ; C, D, 50 \mu \mathrm{m}$. tigation of the influence of the PNS environment and the possible interdependence between oligodendrocytes and astrocytes.

Although the invasion of the nerve roots by central glia occurs in both peripheral glia-depleted and Krox20 null mice, the time courses are different. In the Schwann cell-depleted mice, ectopic oligodendrocytes were already present in the nerve roots from E17.5, whereas in the second case, they were not detected before P3. At least two hypotheses can be formulated to explain this difference. (1) Schwann and BC cells might be both involved in a redundant manner in preventing central glia emigration, with a difference in their modes of action: Krox20 function may not be not required for this function in BC cells, in contrast to Schwann cells. In such a case, in mice depleted of both cell types, migration of central glia occurs after the elimination of Schwann cells, which happens at E15.5E16.5 (data not shown). In contrast, in the Krox20 null mutant, restriction by BC cells is not affected and will still be effective until their normal disappearance at approximately birth (Golding et al., 1997). (2) Alternatively, only Schwann cells might be involved in central glia restriction, but their absence may further facilitate migration within the nerve roots compared with the simple inactivation of Krox20. This does not, however, preclude an indirect involvement of $\mathrm{BC}$ cells, because they give rise to all nerve root Schwann cells in wild-type mice (Maro et godendrocytes are able to efficiently generate CNS-type myelin, with apparently normal characteristics, as shown by the sheath thickness, the organization of nodal and paranodal markers, and the capacity of a single cell to myelinate several axons. In our models, oligodendrocytes are always colocalized with astrocytes, which may suggest their interdependence in terms of survival, migration, and/or differentiation. This latter observation is in agreement with a recent study highlighting the role of astrocytes in the promotion of myelination in the CNS (Sorensen et al., 2008). In our study, ectopic oligodendrocytes and astrocytes were never observed in more distal parts of peripheral nerves, although the DRGs were also colonized (data not shown). This is unlikely to be attributable to a lack of time for migration, resulting from the early death of the mutant mice, because this was also observed in P42 animals carrying a conditional Krox20 mutation. A more likely explanation is that more distal parts of the nerve are or may become unfavorable for CNS glia migration or survival. This possibility should be considered in the light of a recent in vitro study suggesting that NGF-regulated axonal signals inhibit myelination of peripheral axons by oligodendrocytes, whereas they promote myelination by Schwann cells (Chan et al., 2004). Additional experiments will be required to investigate these different possibilities. Of particular interest will be the transplantation of CNS glial cells into the sciatic nerve of wild-type, Krox20 mutant, or Schwann cell-depleted mice, which will allow the inves- al., 2004). Definitive evaluation of the contributions of each cell type to the confinement of central glia will therefore require the specific ablation of BC cells.

Recent work involving time-lapse imaging in the zebrafish showed that Schwann cell depletion led to oligodendrocyte migration along the motor roots of embryos and larvae (Kucenas et al., 2009). Although this study did not include the investigation of the dorsal root and the fate of astrocytes, it is consistent with our observations in the mouse. In addition, Kucenas and collaborators did not observe motoneuron exit from the neural tube parallel to central glia migration in the zebrafish. Consistently, whereas in the Schwann cell-depleted mice ectopic motor neurons were observed in the ventral roots at E12.5, this was not anymore the case at E17.5, when central glia migration has been initiated (supplemental Fig. 2, available at www.jneurosci.org as supplemental material). These data suggest that specific interactions between glia restrict their movement. In addition, together the two analyses suggest that the involvement of peripheral glia in confining the oligodendrocytes to the CNS can be extended to all vertebrates. However, because BC cells may not exist in zebrafish (Kucenas et al., 2009), work performed in this species does not help to evaluate the respective contributions of Schwann and BC cells in the maintenance of the glial cell CNS/PNS boundary in mammals. 
The absence of transgression of this boundary in the case of the Trembler ${ }^{/ J}$ mutant indicates that it is not the presence of myelin that prevents CNS glia immigration into the nerve roots but another Krox20 function, separable from the regulation of myelination. The possible existence of such an additional function is not surprising in view of the intriguing expression of Krox20 in Schwann cells from E15.5, well before the initiation of myelination (Topilko et al., 1994). A key issue will be the identification of the factors that are under Krox20 control and are responsible for the capacity of Schwann or BC cells to maintain CNS glia within the CNS. Such factors might work by segregating peripheral from central glia, and transmembrane or secreted proteins would constitute attractive candidates for performing this function. Indeed, in the case of the confinement of motoneurons by BC cells, such molecules, plexins and semaphorins, have been implicated (Bron et al., 2007; Mauti et al., 2007). Knowledge of a number of Krox20 transcriptional targets (Nagarajan et al., 2001, 2002) should help the identification of the proteins involved in glia segregation.

\section{Implications for human pathology}

The observation of central glia within the nerve roots of a patient affected by congenital peripheral amyelinopathy extends our observation and provides the first example of CNS/PNS boundary transgression in humans. Although the molecular basis of the disease is still being investigated [the coding sequence of KROX20 is not affected (B.F. and J.-M.V., unpublished data)], ultrastructure analysis of the patient's sciatic nerve suggests a block of Schwann cells at the promyelinating stage, a phenotype likely to derive from the observed lack of KROX20 protein. Our observations therefore suggest that the mechanisms responsible for the maintenance of the CNS/PNS boundary might be of similar nature in mice and humans. In this respect, the experiments discussed above and designed to elucidate the basis of the segregation between Schwann cells and central glia will be of particular interest, because their translation to humans might provide the basis for future applications in human pathology. This might be the case for PNS disorders such as chronic inflammatory demyelinating polyradiculoneuropathy (Burns, 2004). This potentially disabling immune-mediated disease results in multifocal inflammation and demyelination of peripheral nerves predominating in nerve roots. Some cases do not satisfactorily respond to standard immunomodulatory therapies and might be accessible to a regenerative treatment aimed at remyelinating nerve roots by central myelin cells. Furthermore, in diseases such as multiple sclerosis, in which oligodendrocytes degenerate, there is a great deal of interest in transplanting Schwann cells into the CNS to form new myelin. The main challenge in this field is directing Schwann cells to migrate through and interact appropriately with CNS cell types. Therefore, understanding the basis of astrocyte, oligodendrocyte, and Schwann cell interactions is a critical issue for regenerative medicine, and we believe that the present work is an important step in this direction. In particular, our data raise the possibility of manipulating Krox20 or one of its targets to avoid astrocyte/Schwann cell segregation, without affecting Schwann cells myelination potential. Finally, although we do not yet know what are the physiological consequences of CNS/PNS transgression per se, our observation of a human case suggests that CNS/PNS transgression is likely to be found in other human peripheral neuropathies and that it may help in their classification. Therefore, it should be searched for systematically when appropriate samples are available.

\section{References}

Ahn K, Mishina Y, Hanks MC, Behringer RR, Crenshaw EB 3rd (2001) BMPR-IA signaling is required for the formation of the apical ectodermal ridge and dorsal-ventral patterning of the limb. Development 128:44494461.

Altman J, Bayer SA (1984) The development of the rat spinal cord. Adv Anat Embryol Cell Biol 85:1-164.

Bermingham JR Jr, Scherer SS, O’Connell S, Arroyo E, Kalla KA, Powell FL, Rosenfeld MG (1996) Tst-1/Oct-6/SCIP regulates a unique step in peripheral myelination and is required for normal respiration. Genes Dev 10:1751-1762.

Berthold CH, Carlstedt T (1977) Observations on the morphology at the transition between the peripheral and the central nervous system in the cat. V. A light microscopical and histochemical study of S1 dorsal rootlets in developing kittens. Acta Physiol Scand Suppl 446:73-85.

Bron R, Vermeren M, Kokot N, Andrews W, Little GE, Mitchell KJ, Cohen J (2007) Boundary cap cells constrain spinal motor neuron somal migration at motor exit points by a semaphorin-plexin mechanism. Neural Dev $2: 21$.

Burns TM (2004) Chronic inflammatory demyelinating polyradiculoneuropathy. Arch Neurol 61:973-975.

Chan JR, Watkins TA, Cosgaya JM, Zhang C, Chen L, Reichardt LF, Shooter EM, Barres BA (2004) NGF controls axonal receptivity to myelination by Schwann cells or oligodendrocytes. Neuron 43:183-191.

Chavrier P, Zerial M, Lemaire P, Almendral J, Bravo R, Charnay P (1988) A gene encoding a protein with zinc fingers is activated during G0/G1 transition in cultured cells. EMBO J 7:29-35.

Coulpier F, Le Crom S, Maro GS, Manent J, Giovannini M, Maciorowski Z, Fischer A, Gessler M, Charnay P, Topilko P (2009) Novel features of boundary cap cells revealed by the analysis of newly identified molecular markers. Glia 57:1450-1457.

Decker L, Desmarquet-Trin-Dinh C, Taillebourg E, Ghislain J, Vallat JM, Charnay P (2006) Peripheral myelin maintenance is a dynamic process requiring constant Krox20 expression. J Neurosci 26:9771-9779.

Duncan ID, Hoffman RL (1997) Schwann cell invasion of the central nervous system of the myelin mutants. J Anat 190:35-49.

Fex Svenningsen A, Colman DR, Pedraza L (2004) Satellite cells of dorsal root ganglia are multipotential glial precursors. Neuron Glia Biol 1:85-93.

Friede RL (1986) Relation between myelin sheath thickness, internode geometry, and sheath resistance. Exp Neurol 92:234-247.

Ghislain J, Desmarquet-Trin-Dinh C, Gilardi-Hebenstreit P, Charnay P, Frain M (2003) Neural crest patterning: autoregulatory and crestspecific elements co-operate for Krox20 transcriptional control. Development 130:941-953.

Giambonini-Brugnoli G, Buchstaller J, Sommer L, Suter U, Mantei N (2005) Distinct disease mechanisms in peripheral neuropathies due to altered peripheral myelin protein 22 gene dosage or a Pmp22 point mutation. Neurobiol Dis 18:656-668.

Golding J, Shewan D, Cohen J (1997) Maturation of the mammalian dorsal root entry zone: from entry to no entry. Trends Neurosci 20:303-308.

Guest JD, Hiester ED, Bunge RP (2005) Demyelination and Schwann cell responses adjacent to injury epicenter cavities following chronic human spinal cord injury. Exp Neurol 192:384-393.

Henry EW, Sidman RL (1988) Long lives for homozygous trembler mutant mice despite virtual absence of peripheral nerve myelin. Science 241:344-346.

Ille F, Atanasoski S, Falk S, Ittner LM, Märki D, Büchmann-Møller S, Wurdak H, Suter U, Taketo MM, Sommer L (2007) Wnt/BMP signal integration regulates the balance between proliferation and differentiation of neuroepithelial cells in the dorsal spinal cord. Dev Biol 304:394-408.

Itoyama Y, Webster HD, Richardson EP Jr, Trapp BD (1983) Schwann cell remyelination of demyelinated axons in spinal cord multiple sclerosis lesions. Ann Neurol 14:339-346.

Jessen KR, Mirsky R (2005) The origin and development of glial cells in peripheral nerves. Nat Rev 6:671-682.

Kucenas S, Wang WD, Knapik EW, Appel B (2009) A selective glial barrier at motor axon exit points prevents oligodendrocyte migration from the spinal cord. J Neurosci 29:15187-15194.

Maro GS, Vermeren M, Voiculescu O, Melton L, Cohen J, Charnay P, Topilko P (2004) Neural crest boundary cap cells constitute a source of neuronal and glial cells of the PNS. Nat Neurosci 7:930-938.

Mauti O, Domanitskaya E, Andermatt I, Sadhu R, Stoeckli ET (2007) 
Semaphorin6A acts as a gate keeper between the central and the peripheral nervous system. Neural Dev 2:28.

Nagarajan R, Svaren J, Le N, Araki T, Watson M, Milbrandt J (2001) EGR2 mutations in inherited neuropathies dominant-negatively inhibit myelin gene expression. Neuron 30:355-368.

Nagarajan R, Le N, Mahoney H, Araki T, Milbrandt J (2002) Deciphering peripheral nerve myelination by using Schwann cell expression profiling. Proc Natl Acad Sci U S A 99:8998-9003.

Peters A, Palay SL, Webster H (1991) The fine structure of the nervous system, Ed 3. Oxford: Oxford UP.

Pietri T, Eder O, Blanche M, Thiery JP, Dufour S (2003) The human tissue plasminogen activator-Cre mouse: a new tool for targeting specifically neural crest cells and their derivatives in vivo. Dev Biol 259:176-187.

Richardson WD, Kessaris N, Pringle N (2006) Oligodendrocyte wars. Nat Rev 7:11-18.

Sham MH, Vesque C, Nonchev S, Marshall H, Frain M, Gupta RD, Whiting J, Wilkinson D, Charnay P, Krumlauf R (1993) The zinc finger gene Krox20 regulates HoxB2 (Hox2.8) during hindbrain segmentation. Cell 72:183-196.

Sherman DL, Brophy PJ (2005) Mechanisms of axon ensheathment and myelin growth. Nat Rev 6:683-690.

Sidman RL, Cowen JS, Eicher EM (1979) Inherited muscle and nerve diseases in mice: a tabulation with commentary. Ann NY Acad Sci 317:497-505.

Sorensen A, Moffat K, Thomson C, Barnett SC (2008) Astrocytes, but not olfactory ensheathing cells or Schwann cells, promote myelination of CNS axons in vitro. Glia 56:750-763.

Soriano P (1999) Generalized lacZ expression with the ROSA26 Cre reporter strain. Nat Genet 21:70-71.

Srinivas S, Watanabe T, Lin CS, William CM, Tanabe Y, Jessell TM, Costantini F (2001) Cre reporter strains produced by targeted insertion of EYFP and ECFP into the ROSA26 locus. BMC Dev Biol 1:4.

Suter U, Welcher AA, Ozcelik T, Snipes GJ, Kosaras B, Francke U, BillingsGagliardi S, Sidman RL, Shooter EM (1992) Trembler mouse carries a point mutation in a myelin gene. Nature 356:241-244.

Svaren J, Meijer D (2008) The molecular machinery of myelin gene transcription in Schwann cells. Glia 56:1541-1551.

Svenningsen AF, Colman DR, Pedraza L (2004) Satellite cells of dorsal root ganglia are multipotential glial precursors. Neuron Glia Biol 1:85-93.

Taillebourg E, Buart S, Charnay P (2002) Conditional, floxed allele of the Krox20 gene. Genesis 32:112-113.

Topilko P, Schneider-Maunoury S, Levi G, Baron-Van Evercooren A, Chennoufi AB, Seitanidou T, Babinet C, Charnay P (1994) Krox-20 controls myelination in the peripheral nervous system. Nature 371:796-799.

Vermeren M, Maro GS, Bron R, McGonnell IM, Charnay P, Topilko P, Cohen J (2003) Integrity of developing spinal motor columns is regulated by neural crest derivatives at motor exit points. Neuron 37:403-415.

Voiculescu O, Charnay P, Schneider-Maunoury S (2000) Expression pattern of a Krox-20/Cre knock-in allele in the developing hindbrain, bones, and peripheral nervous system. Genesis 26:123-126. 